\title{
Adrenaline can restart the heart, but is no good for the brain
}

\author{
Rob Cook clinical director ${ }^{1}$, Peter Davidson clinical adviser ${ }^{2}$, Rosie Martin clinical specialist ${ }^{1}$, on \\ behalf of NIHR Dissemination Centre
}

${ }^{1}$ Bazian, Economist Intelligence Unit healthcare, London, UK; ${ }^{2}$ Wessex Institute, University of Southampton, Southampton, UK

\begin{abstract}
The study

A randomised trial of epinephrine in out-of-hospital cardiac arrest

Perkins GD, Ji C, Deakin CD, Quinn T, Nolan JP, Scomparin C, Regan S, Long J, Slowther A, Pocock H, Black JJM, Moore F, Fothergill RT, Rees N, O'Shea L, Docherty M, Gunson I, Han K, Charlton K, Finn J, Petrou S, Stallard N, Gates S, Lall R for the PARAMEDIC2 Collaborators Published on 18 July 2018 N Engl J Med 2018;379:711-21.

This project was funded by the National Institute for Health Research HTA Programme (project number 12/127/126).

To read the full NIHR Signal, go to: https://discover.dc.nihr.ac.uk/content/ signal-000639/adrenaline-can-restart-the-heart-but-is-no-good-for-thebrain
\end{abstract}

\section{Why was this study needed?}

Adrenaline (epinephrine) injections have been commonly used during cardiopulmonary resuscitation (CPR) for cardiac arrest for more than 60 years, without clear evidence if they are helpful or harmful. Adrenaline can increase the likelihood that the heart will regain a normal rhythm as it directs blood flow to the heart. However, it also causes constriction of small blood vessels, which can reduce blood flow to other organs-including the brain-and may lead to neurological damage.

Use of adrenaline has been linked to better short term survival in observational studies, so it has remained in the cardiac arrest guidelines. Randomised controlled trials have been needed to determine if the benefits outweigh the potential harms. This study assessed routine adrenaline use in cardiac arrests occurring outside hospital.

\section{What did this study do?}

The PARAMEDIC2 randomised controlled trial allocated 8014 people with cardiac arrest (average age 70) to receive either 1 $\mathrm{mg}$ adrenaline or placebo saline injections as part of advanced
CPR treatment by paramedics at the scene. On average, people in the adrenaline group received a total dose of $4.9 \mathrm{mg}$.

In 59\% of each group, bystanders performed CPR. Ambulances took just over six minutes to arrive and paramedics gave the injections on average 21 minutes after emergency services were first called. Major outcomes were adjusted for variations in these factors.

More than one third of patients had unwitnessed cardiac arrest, more than half had no heart electrical activity (asystole), and only $19 \%$ had a shockable rhythm. This may have contributed to the low survival rates.

This large, well designed trial provides much needed UK based, high quality evidence to inform practice.

\section{What did it find?}

- People who received adrenaline had a slightly higher rate of survival at 30 days, 130/4105 (3.2\%) compared with 94/3999 $(2.4 \%)$ who received the saline placebo (adjusted odds ratio $1.47,95 \%$ confidence interval 1.09 to 1.97 ).

- Return of spontaneous circulation was much more likely with adrenaline, occurring in 1457 (36.3\%) people in the adrenaline group versus 468 (11.7\%) people in the placebo group. A substantially higher number of people given adrenaline survived until hospital admission, 947 (23.8\%) compared with 319 (8\%) of the placebo group.

- Although the overall rate of survival at 30 days was slightly better with adrenaline, 39/126 (31\%) people had severe neurological disability in the adrenaline group, compared with $16 / 90(17.8 \%)$ in the placebo group. Severe neurological disability was defined as a score of 4 or 5 on the modified Rankin scale.

- Only 27 people in total had no neurological symptoms at discharge. There were similar numbers of people in each group when combining those with no symptoms, mild or moderate 
neurological disability, modified Rankin scale 0 to 3 (odds ratio $1.19,95 \%$ confidence interval 0.85 to 1.68 ).

\section{What does current guidance say on this issue?}

The 2015 Resuscitation Council UK guideline recommends giving adrenaline every three to five minutes during CPR if a normal heart rhythm is absent. Guidance on the early management of people who have had a cardiac arrest by the Faculty of Pre-Hospital Care at the Royal College of Surgeons of Edinburgh (2017) emphasises the importance of recognising that the person is having a cardiac arrest and beginning CPR as soon as possible.

\section{What are the implications?}

Adrenaline improved the return of spontaneous circulation and likelihood of survival to reach hospital, but only slightly increased survival rates at 30 days. More of those survivors had severe neurological problems.

It remains unclear if out-of-hospital protocols should change as a result of this trial. The findings are also not able to inform hospital cardiac arrest protocols, as use of adrenaline typically occurs within three minutes of cardiac arrest.

Other studies of cardiac arrest taking place out of hospital have shown better outcomes from prompt recognition, bystander $\mathrm{CPR}$, and defibrillation. Strategies to increase public training in CPR and make more defibrillators available may increase the number of people surviving out-of-hospital cardiac arrest.

The overall survival rate is low, so the jury is still out on whether adrenaline should be reserved for use in certain heart rhythms or within a particular time period and not for others.

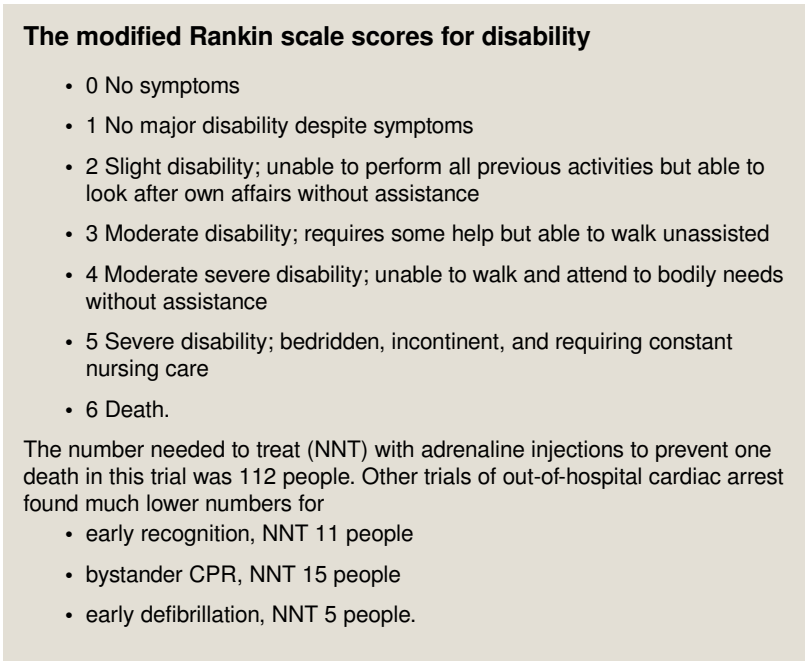

\section{Education into practice}

Are you comfortable with managing cardiac arrest out of hospital? Do you know where the nearest defibrillators are to your places of work? Have you checked the emergency drugs, including adrenaline, where you work and do you know the protocols for administering them?

Competing interests The $B M J$ has judged that there are no disqualifying financial ties to commercial companies. The authors declare the following other interests: none.

Further details of the BMJ policy on financial interests is here: https://www.bmj. com/about-bmj/resources-authors/forms-policies-and-checklists/declarationcompeting-interests

Contributors All authors contributed to development and review of this summary, as part of the wider NIHR Signals editorial team (https://www.bmj.com/NIHRsignals). $R C$ is guarantor.

Disclaimer NIHR Signals are owned by the Department of Health and Social Care and are made available to the BMJ under licence. NIHR Signals report and comment on health and social care research but do not offer any endorsement of the research. The NIHR assumes no responsibility or liability arising from any error or omission or from the use of any information contained in NIHR Signals.

Permission to reuse these articles should be directed to disseminationcentre@nihr.ac.uk.

1 Deakin C, Brown S, Jewkes F, etal . Prehospital resuscitation. London. Resuscitation Council, 2015 https://www.resus.org.uk/resuscitation-guidelines/prehospital-resuscitation. Monsieurs KG, Nolan JP, Bossaert LL, etal. ERC Guidelines 2015 Writing Group. European Resuscitation Council Guidelines for Resuscitation 2015: Section 1. Executive summary. Resuscitation 2015;95:1-80. 10.1016/j.resuscitation.2015.07.038 26477410

3 Resuscitation Council (UK). Resuscitation to recovery. London: Resuscitation Council (UK). 2017. https://www.resus.org.uk/publications/resuscitation-to-recovery

4 Soar J, Deakin C, Lockey A, etal. Adult advanced life support. London. Resuscitation Council, 2015, https://www.resus.org.uk/resuscitation-guidelines/adult-advanced-lifesupport.

Published by the BMJ Publishing Group Limited. For permission to use (where not already granted under a licence) please go to http://group.bmj.com/group/rights-licensing/ permissions 